\title{
EL DOBLE CONFLICTO DE DECISIÓN DE HEISENBERG
}

\section{TWO DECISIONAL CONFLICTS IN HEISENBERG}

\author{
Francisco Augusto Laca Arocena ${ }^{l}$ \\ flarocena@gmail.com
}

\begin{abstract}
Resumen
Se describe, por una parte, el doble conflicto de decisión que se presenta cuando el individuo desea y simultáneamente no desea seguir un curso de acción, en el físico Werner Heisenberg. Conflicto respecto a las decisiones de exiliarse o permanecer en la Alemania nazi y respecto a en qué grado colaborar científicamente con el régimen nazi en la construcción de una bomba atómica. Por otra parte, se propone a discusión el hecho de que en el verano de 1945 mientras Oppenheimer era celebrado por su éxito en coordinar el proyecto Manhattan que posibilitó la destrucción atómica de Hiroshima y Nagasaki, Heisenberg permanecía detenido por su colaboración científica con el régimen nazi aún cuando ésta fuera parsimoniosa y limitada. Planteándose con ello el problema moral, pero también práctico de la relación entre la ciencia y el poder, cuando los científicos obligados a servir a este son juzgados posteriormente según las victorias o derrotas de dicho poder que personalmente no les son imputables.

Palabras clave: conflicto de decisión, ciencia y poder, cultura alemana.
\end{abstract}

\begin{abstract}
A decision conflict is present when a person wants and, simultaneously, not wants to follow an action course. This paper describes firstly the double decision conflict on the physicist Werner Heisenberg: Conflict regarding decisions of going into exile or to remain in the Nazi Germany, and with regard to the degree of scientific collaboration with the Nazi regime to create an atomic bomb. Secondly, discusses the fact that on the summer of 1945 while Oppenheimer was celebrated by his success in coordinating the Manhattan project, which made possible the atomic destruction of Hiroshima and Nagasaki, Heisenberg was in detention for his scientific collaboration with the Nazi regime even when the collaboration was parsimonious and limited. Finally, the Author discusses the ethical and practical problem of the relation between science and power: when scientists forced to serve power are judged lately according to victories and defeats of such power that personally are not imputed to them.
\end{abstract}

Keywords: Decision Conflict, Science and Power, German Culture

1 Universidad de Colima (México). 


\section{La ciencia y el poder: un conflicto moderno}

Hace sesenta años, en los inicios de la era nuclear, Schroedinger (2009) reconocía que en estos tiempos de alta especialización que obligan al esfuerzo de asimilar la mayor cantidad posible de conocimiento en un ámbito muy específico, para después intentar incrementarlo con el trabajo personal, resultaba natural que, inmerso en semejante actividad especializada, el investigador se aislase al olvidar las consecuencias últimas de lo que estaba haciendo. Olvidar las consecuencias de la investigación en ámbitos como la energía atómica o la ingeniería genética pudiera acarrear imprevisibles e irreversibles efectos sobre la vida en este planeta. En la historia de los orígenes de la energía atómica y su apropiación por los gobiernos como arma de destrucción masiva, destaca por la complejidad de sus relaciones con el poder el caso del alemán Heisenberg (1901-1976), también el del estadounidense Oppenheimer (1904-1967). Compartieron ambos la circunstancia de estar al servicio de sus gobiernos en tiempos de guerra total, situación límite para un científico, cuando la línea divisoria entre las exigencias del poder y el horror inaceptable se desdibuja. El primero encarna la ambigüedad forzosa para quien, trabajando en un estado totalitario, la Alemania nazi, encuentra en la gran dificultad de la tarea un posible refugio al conflicto entre su indiscutido patriotismo y los temores de contribuir a aumentar el horror. Las situaciones ambiguas no facilitan salidas elogiables porque siempre dejarán lugar a la polémica de que se podría haber actuado de otro modo. Consecuentemente, quizás injustamente, Heisenberg es una figura cuestionada; sospechoso de ambigüedad ante el régimen nazi, aunque la afortunada consecuencia final de tal ambigüedad fuera que Hitler nunca estuvo cerca de obtener una bomba atómica. Por el contrario, para el Oppenheimer responsable del Proyecto Manhattan que cumplió a satisfacción el encargo de poner las primeras bombas atómicas de la historia en las manos del presidente de su país en 1945, todo fueron elogios y dignidades. Es justo reconocer que hubo un Oppenheimer posterior a las bombas que destruyeron Hiroshima y Nagasaki. Cuando unos años después, durante la paranoia anticomunista del McCarthismo, se convierta en uno de los principales obstáculos en el camino de su colega Teller hacia la obtención de la bomba de hidrógeno, provocará las iras del poder; un poder democrático, pero atemorizado y por ello perseguidor y punitivo. Pensar que los gobiernos democráticos se han mostrado, según Cornwell (2005): "deseosos de actuar con integridad moral en la financiación, administración y utilización de la ciencia y la tecnología es ciertamente una ingenuidad". Todo serán sospechas, acoso y desprestigio para el Oppenheimer de 1954, como todo habían sido elogios y primeras portadas para el de 1945. En la historia de la filosofía política, desde Hobbes y Locke a Isaiah Berlin, pasando por Stuart Mill, Russell y otros, el conflicto entre la libertad de los ciudadanos comunes y las exigencias del poder ha sido un tema constante. El conflicto entre la ciencia, o siendo más modestos, entre algunos científicos y el poder es mucho más reciente, no surge hasta avanzado el pasado siglo en que algunos resultados del progreso científico se tornan apetecibles para el poder; al tiempo que el elevado coste de la investigación moderna obliga a los científicos a recabar financiamiento de los gobiernos y de la gran industria, condicionando con ello la dirección de sus investigaciones y la utilización de sus resultados.

Situándonos en aquél verano de 1945, proponemos a discusión el hecho de que mientras Oppenheimer recibía aplausos y honores de los vencedores por su exitosa coordinación del Proyecto Manhattan; su colega Heisenberg permanecía detenido junto con otros científicos alemanes en Gran Bretaña, sospechoso de ambigüedad ante el régimen nazi, aunque, repitámoslo, la afortunada consecuencia para los propios aliados de aquella ambigüedad fue que Hitler no dispusiera de su bomba atómica. Si finalmente el "juicio histórico" sobre la relación de un científico con el poder, más allá de las decisiones tomadas por el primero, dependiera de algo tan arbitrario y ajeno como el resultado final de unas batallas, algo importante hemos extraviado en nuestras capacidades de juicio y en nuestro desarrollo ético. 


\section{Verano de 1939: ¡es factible un arma atómica?}

Al amanecer del 1 de septiembre de 1939, Hitler invade Polonia; dos días después, el domingo 3 de setiembre Londres y Paris declaraban la guerra a Berlín. Se iniciaba la Segunda Guerra Mundial. Apenas dos semanas antes, tras una visita del físico húngaro Leo Szilard, quien le alertó sobre la probabilidad de que la Alemania nazi estuviera trabajando en la liberación de energía atómica a partir de la fisión del uranio con fines bélicos, una hipótesis verosímil en aquél momento, Einstein había escrito el 15 de agosto una carta al presidente Franklin D. Roosevelt. En aquella misiva, que algunos sugieren fue escrita por el propio Szilard y limitando a Einstein a firmarla, se alertaba al presidente de Estados Unidos sobre las perspectivas de un arma atómica alemana. Inicialmente, la carta no provocó ninguna iniciativa de la administración estadounidense más allá de consultar a expertos. En 1939, y todavía hasta bien entrado 1941, Roosevelt, conocedor de la voluntad aislacionista de su país, no consideraba seriamente una guerra contra Alemania. Algunos han querido atribuir a aquel gesto de Einstein, al alertar a las autoridades estadounidenses, una cierta responsabilidad inicial en que la humanidad entrase en la pesadilla nuclear. El propio Einstein, de cuya militancia pacifista abundan las evidencias (Laca, 2009), nunca se culpó a sí mismo por haber escrito al presidente estadounidense. El riesgo de permitir que los nazis fueran los primeros en obtener un arma atómica parecía inasumible. ¿En aquel verano de 1939 era este un riesgo real?

A comienzos del siglo, el alemán Max Planck y el británico Ernest Rutherford habían dado un fuerte impulso al estudio del átomo. El primero, descubriendo en 1901 que los objetos calientes absorben y emiten radiaciones en cantidades discretas y no en flujo continuo como se pensaba, llamó quantas a estos "paquetes" de energía discreta y con ello estaba dando un nombre a la naciente física cuántica. El segundo zanjó en 1913 las polémicas en torno a la conformación del átomo y propuso un modelo como analogía del sistema solar, lo extremadamente pequeño funcionaría como lo extremadamente grande.
Estableció que el átomo tendría un núcleo muy pequeño respecto al volumen total. Las especulaciones sobre la posible partición de ese núcleo y los efectos de ello marcarán tres décadas de investigaciones nucleares. Alumno de Rutherford en Manchester después de doctorarse en su Dinamarca natal, Niels Bohr fue un decisivo aglutinador de jóvenes físicos que durante las décadas de los veinte y treinta acudían a su instituto de física en Copenhague. Heisenberg fue un asiduo visitante. En 1927, Bohr y un muy joven Heisenberg elaboraron lo que se ha llamado interpretación de Copenhague de la física cuántica, interpretación que sigue vigente en la actualidad (Segré, 2010). En 1932, el premio Nobel le acreditaba como un físico de primer nivel cuando apenas contaba 31 años. En el otoño de 1938, Otto Hanh y su colaborador Strassmann trabajando en el Instituto Kaiser Guillermo en Alemania obtuvieron la fisión de isótopos de uranio U-235 bombardeándolo con neutrones lentos. Parecía que el U-235 (peso atómico 92) se había partido, fisionado, produciendo bario (peso atómico 56). Las transformaciones de un elemento a otro conocidas hasta entonces producían un elemento muy cercano al primero en la tabla periódica, nunca tan distante como el bario del uranio. Dudando de posibles errores en su experimento, Hahn los compartió en carta a su antigua colaboradora y amiga la física Lise Meitner entonces refugiada en Suecia (como tantos científicos judíos, había tenido que emigrar de la Alemania nazi). Meitner opinó que Hahn era un químico demasiado bueno para cometer errores en sus experimentos (Sánchez, 2011). Su sobrino Otto Frish, que estaba presente cuando leía la carta de Hahn, comunicó pocos días después a Bohr en Copenhague este experimento. Aún cuando Bohr quería guardar secreto de la réplica que Meitner y Frish hacían del experimento de Hahn, no pudo evitarse que por medios indirectos llegase a conocimiento de físicos en Estados Unidos. Fermi habló de ello en una conferencia a físicos estadounidenses en enero de 1939, al mismo tiempo que Hahn y su colaborador Strassman publicaban su experimento, aunque manifestaban dudas sobre sus propios resultados. La noticia llegó también a Oppenheimer, entonces en el Instituto Tecnológico de California, quien se manifestaba en estos términos en 
carta a un colega, según Sánchez (2011): "Muchos puntos están todavía oscuros. ¿De cuantas maneras se rompe el U? ¿Aleatoriamente como se podría esperar, o solamente de ciertas maneras? Y lo más importante de todo, ¿se emiten muchos neutrones de la ruptura?". Esta última cuestión era clave, pues de su respuesta dependía la cantidad de energía liberada por la fisión nuclear y con ello su utilidad como posible fuente de energía.

En el último verano de la paz, Heisenberg realizó una gira a Estados Unidos para mantener debates con otros físicos; entre ellos con Oppenheimer en la universidad de Chicago. Recibió invitaciones, incluso amistosas presiones, de algunos colegas como Einstein y Fermi, también de quien fuera su discípulo Teller, para que permaneciese en Estados Unidos a donde ellos habían emigrado de una Europa Central crecientemente antisemita (Einstein y Teller eran judíos). Es oportuno mencionar aquí que Heisenberg siempre mostró desagrado por el antisemitismo de la Alemania nazi y defendió a los "físicos judíos", esto lo reconocen hasta quienes, como Rose (1998), han escrito libros nada benevolentes hacia el físico alemán ¿Estos requerimientos para que no regresase a Alemania estaban motivados exclusivamente por la amistad, o habría también un comprensible temor a que un físico de la talla de Heisenberg fuera utilizado por Hitler? Fermi le habló claramente respecto a que el proceso de fisión nuclear descubierto por Hanh podría ser utilizado para producir una reacción en cadena que liberase grandes cantidades de energía que, teóricamente al menos, podría ser utilizada como una poderosa arma de destrucción. En una guerra que veía ya inevitable, los gobiernos conocedores de esta posible nueva arma impulsarían la investigación con ese objetivo. Los físicos atómicos, le insistió Fermi, se verán obligados por los gobiernos de sus países a colaborar en el desarrollo de un arma semejante (Heisenberg, 2004). Respondiéndonos al interrogante que encabezaba este apartado, en el verano de 1939, los físicos eran conscientes de la posibilidad teórica de obtener una gran cantidad de energía y provocar una reacción en cadena por fisión nuclear del uranio-235; que en la práctica esto fuera factible dependería de muy costosas investigaciones que solo podrían realizarse con fuertes presupuestos gubernamentales. La mencionada carta de Einstein al presidente Roosevelt buscaba urgir al gobierno estadounidense a promover tales investigaciones. Heisenberg sabía, pues en aquellas semanas de gira académica por Estados Unidos, pocas semanas antes del inicio de la guerra en Europa, que la fisión nuclear era posible y que ésta podría provocar una reacción en cadena liberadora de una gran energía que podría utilizarse como un arma de efectos inimaginables hasta ese momento. La primera reacción en cadena no se logrará hasta diciembre de 1942 por el equipo de Fermi en la Universidad de Chicago. No podía ignorar tampoco, como se lo advirtiera su amigo Fermi, que de estallar una guerra que ya parecía inminente, los gobiernos implicados en la contienda demandarían de sus físicos el logro de tal arma, con el argumento difícil de resistir de que muy probablemente el enemigo ya trabajaba en ello.

\section{Heisenberg: primer conflicto de decisión}

En su acepción más elemental, la decisión es la elección de una alternativa cuando se dispone al menos de dos. El supuesto de la racionalidad, que desde la teoría de la elección racional equipara la misma al egoísmo, prescribe que los individuos hacen una elección racional cuando eligen aquella alternativa que previsiblemente les acerque más a sus fines. La racionalidad no tiene que ver con la elección de fines. sino con la adecuación de los medios elegidos para alcanzar los fines que fueran. Desde la psicología cognitiva, se vienen describiendo diferentes sesgos que muestran la imposibilidad práctica de que los individuos satisfagan en la mayoría de sus elecciones, los criterios estrechos de la elección racional (Janis y Mann, 1979; Kahneman y Tversy, 1979, 1984; Laca, 2005, 2012). Hacer elecciones conlleva un conflicto de decisión. Cuando hablamos de conflicto de decisión, de acuerdo con Janis y Mann (1979): "nos estamos refiriendo a tendencias opuestas simultáneas en el individuo a aceptar y rechazar, al mismo tiempo, un determinado curso de acción". Cuando en los primeros días de agosto, poco antes de que Szilard alarmase a Einstein al impulsarle a este a escribir a Roosevelt, Heisenberg embarca en Nueva York; de regreso a Europa, vivía un conflicto de decisión 
en el que se simultaneaban razones para regresar a su patria y otras para aceptar la oferta de quedarse en alguna universidad estadounidense. Conocía la posibilidad teórica de un arma atómica y del previsible interés por esta en gobiernos enfrentados en una guerra. Según escribiría años después, su decisión de regresar a Alemania aquel amenazador verano de 1939 lo motivó su compromiso con un grupo de jóvenes discípulos, con quienes había formado un equipo en el instituto Kaiser Guillermo en Berlín. Heisenberg (2004): "He decidido reunir allí a un grupo de gente joven que quiera participar en la renovación de la ciencia, gente que pueda dedicarse a que haya de nuevo buena ciencia en Alemania después de la guerra. Pienso que cometería una traición si dejara ahora a estos chicos en la estacada". Podrían cuestionarse estas palabras, escritas después de acabada una guerra perdida por Alemania, como una defensa a posteriori de una decisión polémica. La lealtad personal a unos jóvenes discípulos y la lealtad colectiva a su nación, al intentar preservar para ella un buen nivel científico para la posguerra, no son una mala defensa. Justamente, por parecer muy nobles los motivos del regreso a la Alemania nazi, se vuelven sospechosos para quienes quieran verlo así. Muy crítico en su libro sobre Heisenberg y la cultura alemana, Rose (1998) situará en esta decisión de optar por el bando alemán en la guerra que ya se veía inevitable la primera culpa de Heisenberg, previa a su posterior rol en las investigaciones nucleares alemanas.

No son las bombas atómicas el problema moral primario, sino más bien la maldad de la Alemania nazi. Suprimiendo los contextos nazi y aliados Heisenberg crea una falsa simetría entre los presupuesto morales de los científicos aliados y alemanes. Es como si los científicos actuasen en un vacío político, más bien que como luchadores por sus causas respectivas (Rose, 1998:17).

A diferencia de la Primera, la Segunda Guerra Mundial supuso el choque de ideologías incompatibles. El caso de Fritz Haber, premio Nóbel de Química (1918), que siendo judío no sintió conflicto personal alguno en colaborar con la Alemania del Kaiser al coordinar la utilización de gases tóxicos en el campo de batalla, hubiera sido impensable en 1939 debido al antisemitismo nazi. De no haber fallecido previamente, es claro que Haber como Einstein (quien se dolió profundamente del belicismo de Haber en la primera guerra), al igual que Teller, Szilard, Meitner y tantos otros científicos judíos, no hubiera podido permanecer en la Alemania nazi por mera cuestión de supervivencia (fallecería en 1934 exiliado en Gran Bretaña). La guerra que se acercaba aquel verano de 1939 sería en gran parte una guerra ideológica y, desde la perspectiva nazi racial, como lo demostrará además del Holocausto de los judíos el tratamiento inhumano a los civiles soviéticos. Constatando el interés que la Segunda Guerra Mundial continúa provocando en el público, en películas, documentales y libros de continua aparición, la historiadora MacMillan lo atribuye a que aquella guerra fue para una gran mayoría del público, la única guerra buena y moralmente nada ambigua. Según Macmillan (2010): “ Los nazis alemanes, los fascistas italianos, los militaristas japoneses eran gente manifiestamente mala a la que había que derrotar. El hecho de que fuésemos aliados de uno de los mayores tiranos del siglo XX, Josef Stalin, es algo que no se tiene en cuenta”. En ningún otro conflicto aparecerá tan claro para el público de los países aliados cuál era el bando de "los buenos", hasta el punto de olvidar por unos años hasta el inicio de la Guerra Fría, la compañía de Stalin que en cuestión de genocidios no tenía nada que aprender de Hitler. Nos parece ilustrativo, de la gran diferencia entre ambas guerras mundiales, el caso de Bertrand Russell (1872-1970); pacifista militante durante la Primera Guerra Mundial, por lo que fue desposeído de su cátedra de lógica en Cambridge y encarcelado unos meses. Aceptó la participación de la Gran Bretaña en la Segunda Guerra Mundial porque el pacifismo, ante lo que suponía el nazismo, equivaldría a un suicidio colectivo de los europeos (Laca, 2011).

Reconocido el contexto totalitario y racista de aquella Alemania que señala Rose y que por ello no nos parezca moralmente aceptable una simetría entre ambos bandos, en cuyo caso elegir uno u otro sería mera cuestión de patriotismos, ¿supone esto que cualquiera que eligiera quedarse en Alemania elegía el nazismo con todo lo que eso significaba? Heisenberg situará el conflicto de decisión al que se enfrentaban los físicos en 
aquel momento no en la elección de bando como proponen Rose y otros, sino en la decisión de fabricar o no fabricar bombas atómicas para cualquier bando. Situando el problema moral no en la elección de los fines (en este caso la elección de bando), sino en la elección de los medios al servicio de esos fines, Heisenberg (2004) se preguntará años después: “ ¿Se pueden construir bombas atómicas para las causas buenas pero no para las malas? ... ¿Quién decide lo que es bueno o malo? Es bastante fácil establecer que la causa de Hitler y el nacionalsocialismo es mala. Sin embargo, jes la causa americana buena en todos sus aspectos?". Curiosamente, quienes reprochan a Heisenberg quedarse en Alemania no se lo reprochan al también Nobel de física Max Planck, y la razón es que este ya anciano vivía relativamente retirado de la investigación y muy distanciado del régimen, no hubiera sido de ninguna utilidad a los nazis en investigaciones nucleares. Es pues en la posibilidad y voluntad de llevar adelante o no un proyecto de arma atómica donde se situaría el verdadero conflicto de decisión, y no tanto en elegir quedarse o no quedarse en Alemania, algo a lo que una biografía, una lengua y una cultura predisponen en gran medida. Pocos años antes de este decisivo verano de 1939, alarmado ante el control totalitario de la universidad alemana por los nazis, Heisenberg había recabado la opinión de Plank respecto a abandonar Alemania, como veía hacerlo a otros. El anciano físico que había llegado a confrontar personalmente a Hitler en una audiencia argumentando que la expulsión de los judíos de todas las universidades empobrecía muy seriamente la ciencia alemana, vivía retirado en un exilio interior en su casa de Berlín y vigilado por el régimen. Uno de sus hijos será relacionado con la conspiración para matar a Hitler en julio de 1944 y ejecutado. Desistiendo de dar consejos concretos en cuanto a abandonar Alemania o permanecer, Plank opinaba que quienes optaran por permanecer, como era su propio caso, no podrán hacer nada por detener la catástrofe y tendrán que realizar todo tipo de concesiones una y otra vez para sobrevivir. Heisenberg abandonó el domicilio de Plank consciente de las dificultades que se abrían ante su futuro si decidía quedarse en Alemania, recordaría siempre las palabras de Plank "los compromisos que haya que contraer serán reprochados más tarde, con razón y, quizás, castigados. Sin embargo, quizás haya que hacerlo pese a todo". (Heisenberg, 2004). Con frecuencia, las decisiones han de tomarse en situaciones ambiguas en las cuales todas las alternativas parecen simultanear elementos buenos y malos, que ninguna de las alternativas es buena ni mala en todas sus dimensiones (Schick, 2000). La decisión de partir al exilio parece siempre dolorosa, aunque pasados los años uno pudiera alegrarse de ella, pero la decisión de permanecer tampoco es siempre cómoda, en el caso de Heisenberg no parece haberlo sido.

\section{Heisenberg: segundo conflicto de decisión}

Si el primer conflicto de decisión de Heisenberg fue regresar o no a Alemania en agosto de 1939, el segundo será adoptar una actitud proactiva o renuente hacia la fabricación de una bomba atómica para Hitler. A juicio de algunos como Rose (1998), la primera decisión bastaría para condenarle por ponerse a disposición de la Alemania nazi, la segunda decisión sería mero corolario de la primera. Para quienes atiendan a los resultados prácticos de las decisiones y no tanto a la moralidad de las intenciones, el hecho es que Heisenberg no diseñó un proyecto viable para fabricar una bomba nuclear entre 1939 y 1942, año a partir del cual parece haberse desinteresado de una posible bomba atómica que no creía realizable en el tiempo que pudiera durar la guerra.

El problema inicial entre 1939 y 1941 para la obtención de una gran cantidad de energía como la que se necesitaría para fabricar una bomba era determinar la masa crítica o cantidad de uranio necesaria. Desde Fermi, quien en marzo de 1939, consultado por la marina estadounidense, opinó que se necesitarían como 500 kilogramos hasta Teller quien en 1940 calculaba en 30 toneladas la cantidad de uranio necesario, hoy nos sorprende lo desmesurado de aquellas primeras estimaciones (Sánchez, 2011). Otras divergencias importantes entre los mejores físicos de aquel momento fueron respecto a si utilizar neutrones rápidos o lentos para partir el núcleo, y si sería suficiente utilizar uranio 238 presente en la naturaleza, aunque ciertamente no abundante, $o$ 
se requería el U-235 que exigía muy costosos procesos de enriquecimiento. Será en este ambiente de profundas discrepancias en el que hay que situar las dificultades planteadas por Heisenberg al gobierno alemán. En 1940, Otto Frisch exiliado en Inglaterra (sería difícil exagerar hasta qué punto el racismo y el totalitarismo nazi empobrecieron la ciencia en Alemania), partiendo de una formulación avanzada por Pieirls redujo muy considerablemente la cantidad de isótopo U-235 necesaria para producir una reacción en cadena. Frisch (1979): "Para mi asombro era mucho más pequeña de lo que había esperado; no era cuestión de toneladas, sino de algo como una o dos libras". Aunque en la realidad las bombas de Hiroshima y Nagasaki utilizarán una masa crítica superior a lo estimado por Frisch y Pieirls, se había pasado ya a hablar de kilogramos y no de toneladas como en las primeras estimaciones de Bohr, Fermi, Teller y otros en 1939. En el bloqueo de comunicaciones científicas que suponía la guerra, no era posible que Heisenberg trabajando en Berlín tuviera información precisa de hasta qué punto los físicos laborando para Gran Bretaña y Estados Unidos habían rebajado los cálculos respecto al volumen de masa crítica necesaria para una bomba nuclear. Bien comunicado con colegas aliados, Bohr no tuvo noticia de la extraordinaria reducción de uranio necesaria para una masa crítica que habían supuesto los cálculos de Frisch y Pieirls hasta que los aliados lo consideraron definitivamente como uno de los suyos, tras su huida de Dinamarca a Londres en octubre de 1943. Era obviamente imposible que Heisenberg trabajando en territorio enemigo llegase a saberlo. ¿Si lo hubiera sabido, habría cambiado esto su escepticismo hacia una posible bomba atómica en breve tiempo? ¿Hubiera estimado viable la obtención del arma nuclear antes de concluir la guerra y, entonces, hubiera abrazado con entusiasmo el proyecto? Como toda pregunta hipotética referida a supuestos que no ocurrieron, no puede responderse objetivamente, quien aventurase una respuesta a tal cuestión tan solo estaría manifestando su opinión personal sobre el físico alemán.

Tras la primera decisión, regresar a Alemania en agosto de 1939, el segundo conflicto de decisión de Heisenberg parecería ser desde nuestra visión actual aceptar o rehusar colaborar en los encargos que recibiera de las autoridades nazis. Segùn Pérez Gay (2011), el novelista Theodor Fontane había dicho refiriéndose a la Alemania de la segunda mitad del siglo XIX que Prusia no era un país que tuviera un ejército, sino un ejército que tenía un país. La Alemania nazi no era un país con un partido único, era un partido único con un país. Rehusarse a obedecer en aquel contexto totalitario no era una opción para nadie que apreciase su vida y el bienestar de su familia. Una persona tiene una elección que hacer -diremos que enfrenta un problema- sólo cuando tiene opciones entre las cuales elegir (Schick, 2000). El conflicto de decisión de Heisenberg solo podía estar entre la opción de colaborar totalmente y la opción de colaborar con parsimonia y renuencia que es lo que acabaría haciendo; no colaborar no era una opción en aquellas circunstancias.

No tendríamos espacio en un artículo para intentar recopilar los antecedentes históricos inmediatos del totalitarismo que se impuso en Alemania a partir de 1933, los traumáticos efectos de la capitulación de 1918 y las graves convulsiones sociales en la república de Weimar. Tampoco para analizar importantes condicionamientos culturales, la persistencia en Alemania de ciertos fantasmas del romanticismo que nunca arraigaron con esa fuerza en otras latitudes. Diversos autores identificaron en el nazismo la expresión exacerbada de algunos componentes irracionales del romanticismo (Safranski, 2009); tales como la búsqueda de refugio frente a las incertidumbres del presente en un inexistente pasado mitificado, así como una fuente de poder en la tierra y el linaje, "Todo lo que constituye el nazismo está contenido germinalmente en el romanticismo (Klemperer en Safranski, 2009)". Personalmente, Hitler no era un romántico, sus ideas procedían de una biología vulgarizada que se concretaba en un darwinismo social extremo y en racismo (Safranski, 2009), pero la prevalencia de fuertes pulsiones románticas en el pueblo alemán le hizo particularmente receptivo al discurso milenarista del nacionalsocialismo. Solo una entrega ciega y muy mayoritaria del pueblo alemán permitió a Hitler establecer en muy pocos años un estado totalitario como no se había conocido en la Europa moderna. Este carácter totalitario del régimen nazi, que no dejaba ninguna 
actividad profesional fuera de su control, forzó a que personas tan ajenas inicialmente a intereses políticos como cineastas o directores de orquesta se vieran forzosamente asociados al nazismo si querían ejercer sus actividades artísticas; sufriendo por ello, posteriores procesos de "desnazificación" tras la guerra. Si era imposible dirigir una película o una orquesta en Alemania en aquellos años sin la tolerancia del régimen, y no era un régimen proclive a tolerancia alguna con quienes manifestasen la menor disidencia, no digamos oposición, la situación no era diferente en el ámbito de la investigación científica. Heisenberg y colegas como Wizsacker y Hahn no hubieran podido proseguir sus investigaciones sin algún grado de acomodación al régimen, sin aceptar algunos de esos compromisos de los que Plank advirtiera pocos años antes ¿Y qué sentido hubiera tenido quedarse en Alemania para no realizar actividad científica alguna?

\section{Parcialidad del tribunal de la historia y utilitarismo de los vencedores}

En la cultura alemana, en la cual no hay un equivalente liberal del inglés Locke ni de un rebelde como Voltaire, las figuras clave respecto al conflicto obediencia/rebeldía del ciudadano frente al poder son Lutero y Kant. El primero distinguía una libertad interna o de conciencia y una libertad externa, que hoy asimilaríamos a los derechos civiles; temeroso de que los príncipes alemanes, sus mentores, se alarmasen respecto a las reformas ante las rebeliones de campesinos exigiendo derechos civiles. Lutero justificará la represión de los campesinos con palabras terribles. El deber de los buenos cristianos era obedecer al poder temporal del príncipe y permanecer confiados en su libertad interna. Esta acomodación a la obediencia formal al poder temporal, aunque internamente se pudiera estar en profundo desacuerdo con él, que ha caracterizado la vida de muchos bajo diferentes regímenes autoritarios, impregnó la cultura alemana a partir de la Reforma. En el siglo XVIII, el precepto de Lutero de refugiarse en una libertad interna compatible con la obediencia externa a la autoridad será redefinido y en sus consecuencias prácticas confirmado por Kant (2001). El aparente individualismo liberal de los escritos kantianos sobre ética queda debilitado por su filosofía política (Rose, 1998). Estamos ante una renovación kantiana de la dualidad luterana: aunque la ley moral del sujeto racional y autónomo es superior a la ley heterónoma del estado, Kant desautoriza en la práctica al sujeto a desobedecer leyes o rebelarse contra un poder injusto. En su Metafísica de las costumbres (1797), expresa palabras tan duras como las de Lutero contra quienes se rebelen contra la autoridad del estado. Pocas décadas después, esta absolutización del estado alcanzará su punto culminante en Hegel para quien el militarista y conservador estado de Prusia es la culminación de la historia. Describiendo el fracasado atentado contra Hitler del 20 de julio de 1944 y lo extremadamente fácil que fue detener a todos los oficiales implicados, Joachim Fest (2005) en su magistral biografía de Hitler, aludirá a los complejos morales que paralizaban a estos oficiales quienes no lograban desprenderse totalmente de su juramento de fidelidad al führer como comandante en jefe del ejército: "Antes que defenderse a sí mismos, se autoprohibieron mentir, por motivos morales, sin pararse a considerar los peligrosos efectos de su orgullo en manos de los jueces". Refiriéndose a otro grupo de oposición al nazismo encabezado por Moltke, nieto del famoso mariscal, desarticulado en 1943, el periodista William Shirer (2011), durante años corresponsal en Berlín escribirá que: "Moltke y sus amigos tenían el valor de hablar -y por ello fueron ejecutados-, pero no el de actuar". La única oposición real a Hitler vino de estos grupos minoritarios de aristócratas u oficiales, y estos se negaban a sí mismos, la menor oportunidad de éxito paralizados por dudas morales respecto a juramentos y lealtades difícilmente comprensibles desde fuera de la cultura tradicional prusiana a la que muchos de ellos pertenecían, una cultura impregnada de los principios de Lutero y Kant respecto a la obediencia al estado. La respuesta del régimen nazi al atentado del 20 de julio fue brutal contra el ejército de tierra, altos oficiales fueron expulsados del ejército, torturados y ejecutados. La venganza alcanzaría incluso a tres mariscales (Witzleben, von Kluge y Rommel). El ejército en su conjunto aceptó pasivamente todo esto con una sumisión al poder político que solo 
es posible entender recordando la historia alemana, de acuerdo con Shirer (2011): "y según la cual la obediencia ciega a los dirigentes políticos era la más alta virtud del hombre alemán y valoraba el servilismo". Hay que comenzar por entender que Heisenberg se educa en el seno de esta tradición cultural que formaba a las sucesivas generaciones en la creencia de que la rebeldía contra el poder nunca era una opción legítima.

Consideremos el caso del filósofo Heidegger cuya afinidad pública con el nazismo fue mucho más inequívoca que en el caso de Heisenberg. Alguien tan poco sospechoso de condescendencia con el nazismo como Sartre, disculpaba parcialmente en 1944 a Heidegger al atribuir su comportamiento bajo el nazismo al miedo, a su afán por proseguir su carrera académica y al conformismo (Collins, 2004). Miedo y conformismo que el historiador Fritzsche (2011) atribuye a la mayoría de los compatriotas de Heidegger y Heisenberg: "Los alemanes se acercaron a las políticas nazis movidos por el miedo, el oportunismo y la ambición profesional, así como por niveles variantes de convicción ideológica". Miedo, oportunismo, carrera profesional. El miedo es una emoción y, por consiguiente, no imputable a la voluntad del sujeto. Un estado totalitario como el nazi era experto en provocarlo, y la respuesta adaptativa de muchos ante una situación generadora de miedo es el conformismo con la misma. "El miedo consigue todavía mucho más que la violencia, los rumores son más valiosos que los hechos. Lo indeterminado resulta más amenazante" (Junger, 2008. p 21). Para quienes no fueran judíos ni estuvieran en las listas de enemigos políticos, tales como comunistas y socialdemócratas, para el común de los alemanes como Heidegger y Heisenberg, la posibilidad de disentir del régimen iba asociada a consecuencias indeterminadas y por tanto muy amenazantes para sus carreras y sus vidas privadas. "Los alemanes tuvieron que interrogarse sobre la importancia de encajar, la conveniencia de cooperar" (Fritzsche, 2011. p 15).

Una guerra total, como lo fue la Segunda Guerra Mundial, expresa la voluntad de destrucción del enemigo hasta el límite en que este acepte la rendición incondicional. Ante los tribunales de la historia que suelen integrar los vencedores, los excesos que estos hubieran cometido para lograr la victoria, para lograr precisamente convertirse en jueces de los vencidos, son ignorados o exculpados mientras la culpabilidad de los vencidos no admite atenuantes. Un sistema totalitario y racista como lo era la Alemania nazi cometió crímenes contra la humanidad que han sido bien documentados y merecen la condena sin ambigüedades, esto no es cuestionable; ello no supone que los aliados fueran inocentes de todo crimen contra las poblaciones civiles. Desde 1943, las ciudades alemanas fueron bombardeadas sistemáticamente más allá de ningún objetivo militar estratégico por mera voluntad de aterrorizar a la población. En la destrucción de Hamburgo fallecieron más de 50.000 civiles, en la de Dresde, cuando ya Alemania estaba derrotaba, las víctimas civiles no fueron menores de 30.000 en solo una noche. El físico británico Freema Dyson, trabajando durante la guerra como analista para la aviación británica, fue testigo de la voluntad de destruir zonas residenciales de las ciudades alemanas que no suponían ningún objetivo militar. Con escéptica ecuanimidad escribirá, según Pérez (2011): “Después de la guerra entendí que yo era igual a los asesinos burócratas que trabajaban con la máquina de la muerte de Adolf Eichmann. La única diferencia es que ellos terminaron ahorcados o en la cárcel mientras yo salí libre". Por su parte, los soviéticos se calcula que expulsaron 18 millones de alemanes de sus hogares en las regiones orientales de Alemania, obligados a caminar hacia el oeste, mujeres y niños fallecieron de inanición y frío. El mando estadounidense en Europa calculó en tres millones el número de civiles alemanes, sobre todo mujeres y niños, fallecidos en aquél éxodo (Regan, 2006). Alemania había sido brutal en su ocupación del territorio soviético manifestando una voluntad de exterminio de poblaciones civiles, y el ejército soviético actuaría con comparable brutalidad con la población alemana. En la Segunda Guerra Mundial, ninguno de los bandos respetó los más elementales derechos humanos de las poblaciones civiles, no lo habían hecho los vencidos y tampoco sería una preocupación de los vencedores.

En este punto, es inevitable la sospecha de "dos medidas" por parte de los vencedores al juzgar a los científicos que permanecieron en 
Alemania durante la guerra. Científicos alemanes que en la posguerra no tuvieron problema alguno por su pasado político habían mantenido con el régimen nazi una actitud de colaboración mucho mas clara que Heisenberg. El etólogo Konrad Lorenz, premio Nobel de medicina en 1973, muy popular por sus trabajos sobre la impronta de los gansos recién nacidos, ¿quién no ha visto las simpáticas fotografías del viejo investigador caminando seguido de una fila de pequeños gansos?, fue director del Instituto de Psicología Comparada de Könisberg nombrado por el gobierno nazi. De 1940 a 1943, los trabajos de Lorenz estuvieron dedicados a reforzar la ideología nazi (Watson, 2007). Imposible ignorar el caso del ingeniero aeronáutico von Braun, diseñador de las bombas volantes V1 y V2 que se arrojaron sobre Londres, construidas en una fábrica subterránea, Dora, donde miles de trabajadores en condiciones de esclavitud perecieron. Los estadounidenses protegerán a von Braun de toda molestia y lo acomodarán en Estados Unidos encomendándole la dirección del futuro programa espacial de la NASA. Puede deducirse que no teniendo los estadounidenses ningún ingeniero aeronáutico conocedor de la incipiente tecnología de los misiles, necesitaban a von Braun; pero habiendo logrado con éxito la bomba atómica en el proyecto coordinado por Oppenheimer, no tenían ninguna necesidad de los conocimientos de Heisenberg. Los científicos alemanes no fueron peor o mejor tratados por su grado de colaboración con el nazismo, sino por la necesidad que los vencedores tuvieran de sus conocimientos.

Los dos conflictos de decisión afrontados por Heisenberg en 1939: regresar aquel verano a Alemania o permanecer en los Estados Unidos; y qué grado de colaboración científica asumir con el régimen nazi; inevitablemente serán juzgados posteriormente desde la perspectiva de la derrota alemana. La racionalidad de una elección solo puede evaluarse desde las opciones disponibles y la información sobre las que dispusiera el individuo en el momento cuando tuvo que hacer su elección. En el otoño de 1939, nadie podía prever con base en informaciones objetivas en aquel momento cuál sería el resultado final de la guerra que comenzaba. ¿Qué lugar ocuparían en la historia, qué juicio habrían merecido, Oppenheimer y el resto de físicos que ejecutaron el Proyecto Manhattan si los Estados Unidos hubieran perdido aquella guerra?

\section{Referencias bibliográficas}

Collins, J. (2004). Heidegger y los nazis. Barcelona. Gedisa. Col. Encuentros contemporáneos . (Traducción de Heidegger and the Nazis. Cambridge. UK. Icon Books Ltd. 2000).

Cornwell, J. (2005). Los científicos de Hitler. Ciencia, guerra y pacto con el diablo. Barcelona. Paidós. Col. Historia Contemporánea. (Traducción de Hitler's scientists. London. Viking Penguin Books. 2003).

Fest, J. (2005). Hitler. Una biografía. 2 Ed., Barcelona. Planeta. (Traducción española de Hitler. Econ Ullstein List Verlag GmbH \& Co. KG, 2002).

Frisch, O. R. (1979). What litte I remember. Cambridge, GB. Cambridge University Press.

Fritzsche, P. (2011). Vida y muerte en el Tercer Reich. Barcelona. Crítica. (Traducción de Life and death in the Third Reich. Harvard, MA. Harvard College. 2008).

Heisenberg. W. (2004). La parte y el todo. Conversaciones en torno a la física moderna. Castellón, España. Ellago Ediciones. Col. Las Islas. (Traducción de Der teil und ganze).

Janis, I., Mann, L. (1979). Decision making: A psychological analysis of conflict, choice and commitment. New York. The Free Press, a division of MacMillan Inc.

Junger. E. (1994). Sobre la línea. Barcelona. Paidós. Col. Pensamiento contemporáneo $\mathrm{n}^{\circ}$ 28. (Traducción de Uber die Linie. Sttugart. Ernst Klett Verlag. 1956, 1977).

Kant, I. (2001). Basic writtings of Kant. New York. The Random House Publishing.

Laca, F. A. (2005). Elección de estrategias de afrontamiento del conflicto bajo presión de tiempo. Bilbao, España. Editorial Universidad del País Vasco.

Laca, F. A. (2009). Albert Einstein: un sionismo pacifista. Acta Universitaria. Vol. 19(1). 12-20. (ISNN 0186-6266). 
Laca, F. A. (2011). Bertrand Russell: pacifismo político relativo. Convergencia, Revista de Ciencias Sociales. 57. $129-145$. (ISNN 1405-1435).

Laca, F. A. (2012). Racionalidad limitada y gestión de riesgos en la sociedad del riesgo global. RIPS Revista de Investigaciones Políticas y Sociológicas. Vol. 11(1). 105118. (ISSN 1577-239X).

MacMillan, M. (2010). Juegos peligrosos. Usos y abusos de la historia. Barcelona. Ariel. Col. Actual. (Traducción de Dangerous games. The uses and abuses of history. Modern Library. 2010).

Regan, G. (2006). Guerras, politicos y mentiras. Cómo nos engañan manipulando el pasado y el presente. Barcelona. Crítica. (Traducción de Picking the bones. Reclaiming the past from the politicians. Geoffrey Regan 2006).

Rose P. L. (1998). Heisenberg and the Nazi atomic bomb project. A study in German culture. Berkeley, CA. University of California Press.

Overy, R. (2010). Al borde del abismo. Diez días que condujeron a la segunda guerra mundial. México D. F. Tusquet Editores. Col. Tiempo de Memoria. (Traducción de 1939. Countdown to war. London. Penguin Books Ltd. 2009).

Pérez J. M. (2011). La profecía de la memoria. Ensayos alemanes. México D. F. Ediciones Cal y Arena.

Schick, F. (2000). Hacer elecciones. Una reconstrucción de la teoría de la decisión.
Barcelona. Gedisa Editorial. Col. Filosofía. (Traducción de Making choices. A recasting of decision theory. Cambridge University Press. 19997).

Sfranski, R. (2009). Romanticismo. Una odisea del espíritu alemán. Barcelona y México D. F. Tusquet Editores. Col. Tiempo de Memoria, ${ }^{\circ} 75$. (Traducción de Romantik. Eine Deutsche Affäre. Carl Hanser Verlag. Múnich y Viena, 2007).

Sánchez Ron J. M. (2011). El poder de la ciencia. Historia social, política y económica de la ciencia (siglos XIX y XX). Barcelona. Crítica.

Segré, G. (20010). Fausto en Copenhague. Una lucha por el alma de la física moderna. Barcelona. Ariel. (Traducción de Faust in Copenhagen. A struggle for the soul of physics. 2009).

Shirer, W. L. (2011). Auge y caída del Tercer Reich. Vol. II. Guerra y derrota. México D. F. Planeta. (traducción de The rise and fall of the Third Reich. 1961, renewed 1989).

Schrödinger, E. (2009). Ciencia y humanismo. $3^{\mathrm{a}}$ Ed. Barcelona. Tusquets Editores. Col. Metatemas, $N^{\circ} 10$. (Traducción de Science and Humanism. Cambridge University Press. 1951).

Watson. P. (2007). Historia intelectual del siglo XX. Barcelona. Crítica. (Traducción de A terrible beauty. A history of the people and ideas that shaped the modern mind. New York. Harper Collins, 2001). 
